\title{
Morfologia setal de Parastacus brasiliensis (von Martens) (Crustacea, Decapoda, Parastacidae)
}

\author{
Ana Cristina Moura Horn \& Ludwig Buckup
}

Departamento de Zoologia, Universidade Federal do Rio Grande do Sul. Avenida Bento Gonçalves 9500, prédio 43435, sala 219, 91501-970, Porto Alegre, Rio Grande do Sul, Brasil.E-mail: anahorn@ig.com.br; Ibuckup@yahoo.com.br

\begin{abstract}
Setae morphology of Parastacus brasiliensis (von Martens) (Crustacea, Decapoda, Parastacidae). The family Parastacidae comprises the limnic crustaceans popularly known as crayfishes or crawfishes. Parastacus Huxley, 1879 is the only genus occurring in Brazil and has an endemic species, Parastacus brasiliensis (von Martens,1869), in Rio Grande do Sul State. The individuals were collected with traps from a brook in the springs of Gravataí basin, Taquara, Rio Grande do Sul State, Brazil. The captured animals were taken to the Universidade Federal do Rio Grande do Sul Carcinology Laboratory and raised to full growth in aquaria. The specimens were dissected, and the setae analysed under optical microscopy and drawn with the aid of a camera lucida. Material was prepared for photography under scanning electron microscope. The analysis and classification of all types of setae in the adult forms of P. brasilieinsis was performed.
\end{abstract}

KEY WORDS. Crayfish, Neotropical, setae classification.

RESUMO. A familia Parastacidae compreende os crustáceos límnicos popularmente conhecidos como lagostins da água doce. Parastacus Huxley, 1879 é o único gênero que ocorre no Brasil, e inclui, no estado do Rio Grande do Sul, a espécie endêmica, Parastacus brasiliensis (von Martens, 1869). Os espécimes foram coletados com armadilhas em um arroio nas cabeceiras da bacia do Rio Gravataí, município de Taquara, Rio Grande do Sul, Brasil. Os animais capturados foram transportados até o Laboratório de Crustáceos Universidade Federal do Rio Grande do Sul e criados em aquários até atingirem o estado adulto. Os espécimes foram dissecados e suas estruturas examinadas por microscopia óptica e desenhados em câmara clara. O material foi ainda preparado para fotografia sob exame com microscópio eletrônico de varredura. Procedeu-se à análise e classificação de todos tipos de setas encontradas nas formas adultas de P. brasilieinsis.

PALAVRAS CHAVE. Classificação setal, lagostins, neotropical.

\begin{abstract}
A família Parastacidae está representada na América do Sul por espécies de Parastacus Huxley, 1879, Samastacus Rieck, 1971 e Virilastacus Hobbs, 1991. Parastacus brasiliensis (von Martens, 1869) é endêmica da região meridional brasileira, ocorrendo nas bacias que formam o estuário do Guaíba, na depressão central do Estado do Rio Grande do Sul (Buckup 1999). Parastacus brasiliensis é um lagostim que escava suas habitações nas margens úmidas e argilosas de pequenos ambientes lóticos, como arroios, riachos e fontes (Buckup \& Rossi 1980).

Vários autores propuseram sistemas de classificação para as setas encontradas em crustáceos. Entre esses, destaca-se a contribuição de Thomas (1970), que investigou as setas existentes nos apêndices de Austropotamobius pallipes (Lereboullet, 1858), lagostim europeu, propondo um sistema de classificação. FIsH (1972) descreveu as setas de Eurydice pulchra Leach, 1815 (Isopoda) e em seguida FARMER (1974) desenvolveu trabalho semelhante com Nephrops norvegicus (Linnaeus, 1758).
\end{abstract}

DraCh \& JACQUES (1977) também fizeram propostas abordando esquemas gerais de classificação de setas em decápodos.

O primeiro sistema de classificação apoiado na morfologia funcional das setas foi proposto por JACQUES (1989). WATLING (1989) adotou o conceito da homologia das setas para embasar sua proposta de classificação das setas de crustáceos.

No Brasil, poucos trabalhos foram desenvolvidos sobre este tema, com destaque para os trabalhos de Bond-Buckup et al. (1996) com Aegla prado Schmitt, 1942, BuENo \& BOND-Buckup (1996) com Aegla violacea Bond-Buckup \& Buckup, 1994, BondBucKUP et al. (1998), com as espécies Aegla longirostri BondBuckup \& Buckup, 1994, A. ligulata Bond-Buckup \& Buckup, 1994 e A. platensis Schmitt, 1942. CaLazans \& Ingle (1998), também realizaram minucioso trabalho caracterizando os estágios larvais de Pleoticus muelleri Bate, 1888 (Solenoceridae) com base na morfologia das setas.

Vários autores referiram-se à importância das pesquisas

Revista Brasileira de Zoologia 21 (4): 765-768, dezembro 2004 
sobre a morfologia, a classificação e a distribuição dos diversos tipos de setas nos crustáceos. THomas (1970) lembrou a importância do papel das setas na vida dos crustáceos e CaLAZans \& INGLE (1998) destacaram a possibilidade de utilizar tais informações na taxonomia comparativa, em especial nas fases larvais. Nenhum parastacideo sulamericano foi examinado, até esta data, a nível de ultramicroscopia eletrônica, com respeito a sua morfologia setal. Assim, a presente contribuição, pretende oferecer uma contribuição para o conhecimento da forma e da distribuição das setas nos apêndices de uma espécie neotropical de lagostim-de-água-doce, com vistas a seu uso na caracterização das espécies e para estudos evolutivos no gênero.

\section{MATERIAL E MÉTODOS}

Os animais utilizados no presente estudo foram coletados, majoritariamente, nas cabeceiras do Rio Gravataí, mais precisamente na nascente do Arroio Mineiro, na localidade de Fazenda Fialho, município de Taquara $\left(29^{\circ} 46^{\prime} \mathrm{S}, 50^{\circ} 53^{\prime} \mathrm{W}\right)$ e ainda, minoritariamente, em um arroio da bacia do Rio Jacuí, junto ao Morro Cerro Negro, município de Mariana Pimentel

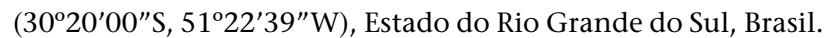

Para a captura dos animais, utilizou-se armadilhas de tubo de PVC com $50 \mathrm{~mm}$ de diâmetro e $20 \mathrm{~cm}$ de comprimento com grade de entrada em arame ou tela de latão, conforme FonTOURA \& Buckup (1989a, b). Para atrair os animais foram colocadas iscas de charque ou fígado de galinha no interior das armadilhas. O funcionamento da armadilha está baseado na portinhola de entrada do tudo plástico, que permite apenas a entrada do animal, travando-se na tentativa de saída.

Sabendo-se por pesquisas anteriores (FONTOURA \& BUCKUP 1989a, b) que Parastacus brasiliensis tem hábitos noturnos, ou seja, sai de suas galerias subterrâneas para deslocar-se pelo corpo d'água em busca de alimento apenas após o pôr-do-sol, as armadilhas foram instaladas no final da tarde, ficando submersas em locais de fluxo d'água para favorecer a expansão dos aromas atrativos das iscas nas armadilhas. Na manhã seguinte, os animais capturados foram recolhidos e trazidos em um recipiente com água do local ao Laboratório de Carcinologia, Departamento de Zoologia, Universidade Federal do Rio Grande do Sul (UFRGS), onde foram mantidos em aquários de cultivo para posterior manipulação.

O exame e a documentação óptica microeletrônica foram implementados com o apoio do Centro de Microscopia Eletrônica, UFRGS. Para análise em microscopia eletrônica de varredura utilizou-se a técnica adaptada de Scotto (1980) e Felgenhauer (1987). Primeiramente, as estruturas dissecadas a serem analisadas foram fixadas em formalina tamponada a $10 \%$. Em seguida foi feita a fixação secundária, onde o material é então lavado com água destilada e fixado novamente em tetróxido de ósmio a $2 \%$ em solução de tampão fosfato por duas horas. Seguiram-se três banhos em água destilada (cinco minutos cada). Para a remoção de tecidos aderidos, colocou-se o material em imersão em $\mathrm{KOH}$ a $15 \%$, por doze horas em temperatura ambiente. A desidratação constituiu-se de três banhos, de cinco minutos cada, em água destilada, e dois banhos de cinco minutos cada em álcool etílico nas concentrações $25,50,70,80,95$ e 100\%. Posteriormente, o material foi imerso em solução de álcool etílico-acetona, na proporção de 1:1 e, após cinco minutos, colocado em acetona pura por mais cinco minutos e logo após feita a secagem em ponto crítico, no aparelho Baltec, modelo CPD 030. A seguir, montou-se o material sobre fita autocolante dupla face em suporte de alumínio. A metalização em ouro foi feita no Sputter Coatter SCD 050 Baltec. Por fim, observou-se o material no microscópio eletrônico de varredura, aparelho de marca Jeol, modelo JSM 5800.

A classificação das setas foi feita conforme THомаs (1970), tendo sido considerados como elementos fundamentais para a determinação dos tipos de setas a presença ou ausência de septo basal, presença ou ausência de anulação e tipo de expansões cuticulares da haste setal.

\section{RESULTADOS}

Em $P$. brasiliensis foram encontradas treze tipos diferentes de setas, que foram assim classificadas: 1) Seta simples (Fig. 1) (sinônimo: "acuminate setae", "rod setae", "papillate setae", segundo Тномаs 1970): desprovida de projeções cuticulares. A anulação é evidente na região mediana da haste ou está situada a $1 / 3$ da base. São setas muito variáveis no comprimento e espessura. Não apresenta septo basal. 2) Seta gancho (Fig. 2) ("Hamate setae" segundo Thомаs 1970): desprovida de projeções cuticulares. Muito pequena quando comparada com outras setas. Não apresenta septo basal. 3) Seta dentiforme (Fig. 3) ("Tooth setae" segundo Тномаs 1970): setas robustas e inseridas obliquamente no eixo longitudinal do segmento. Não apresenta septo basal. 4) Seta cuspidada (Fig. 4): setas robustas, com anulação basal. Não apresentam septo basal. 5) Seta conada (Fig. 5): setas curtas, com anulação basal distinta. 6) Estetasco (Fig. 6): anulação bem evidente no terço proximal e sem septo basal. Parede (cutícula) muito fina na região apical. 7) Seta plumosa (Fig. 7): seta longa com sétulas que se dispõem da base até o ápice, arranjadas em duas fileiras opostas, em ângulo aproximado de $180^{\circ}$. Com septo basal proeminente e anulação difícil de ser visualizada. 8) Seta paposa (Fig. 8): com sétulas dispostas aleatoriamente em torno da haste. O comprimento varia de acordo com a localização. Com septo basal. Anulação difícil de visualizar. 9) Seta serrada (Fig. 9): possuem duas fileiras opostas de dentículos. Anulação evidente e septo basal presente. Grande variedade de tamanhos e formas de dentículos, que podem começar logo após a anulação ou ser apicais, robustos ou não, dependendo da localização da seta. 10) Seta multidenticulada (Fig. 10): mais longa que a serrada. Ocorrem dentículos distantes da anulação, os quais são pequenos e distribuídos em várias fileiras. Dentículos com projeções na parte distal. Anulação evidente na região mediana da haste. 11) Seta plumodenticulada: com sétulas que ocorrem na região pré e pós-anular e dentículos na região apical. Anulação não visível

Revista Brasileira de Zoologia 21 (4): 765-768, dezembro 2004 

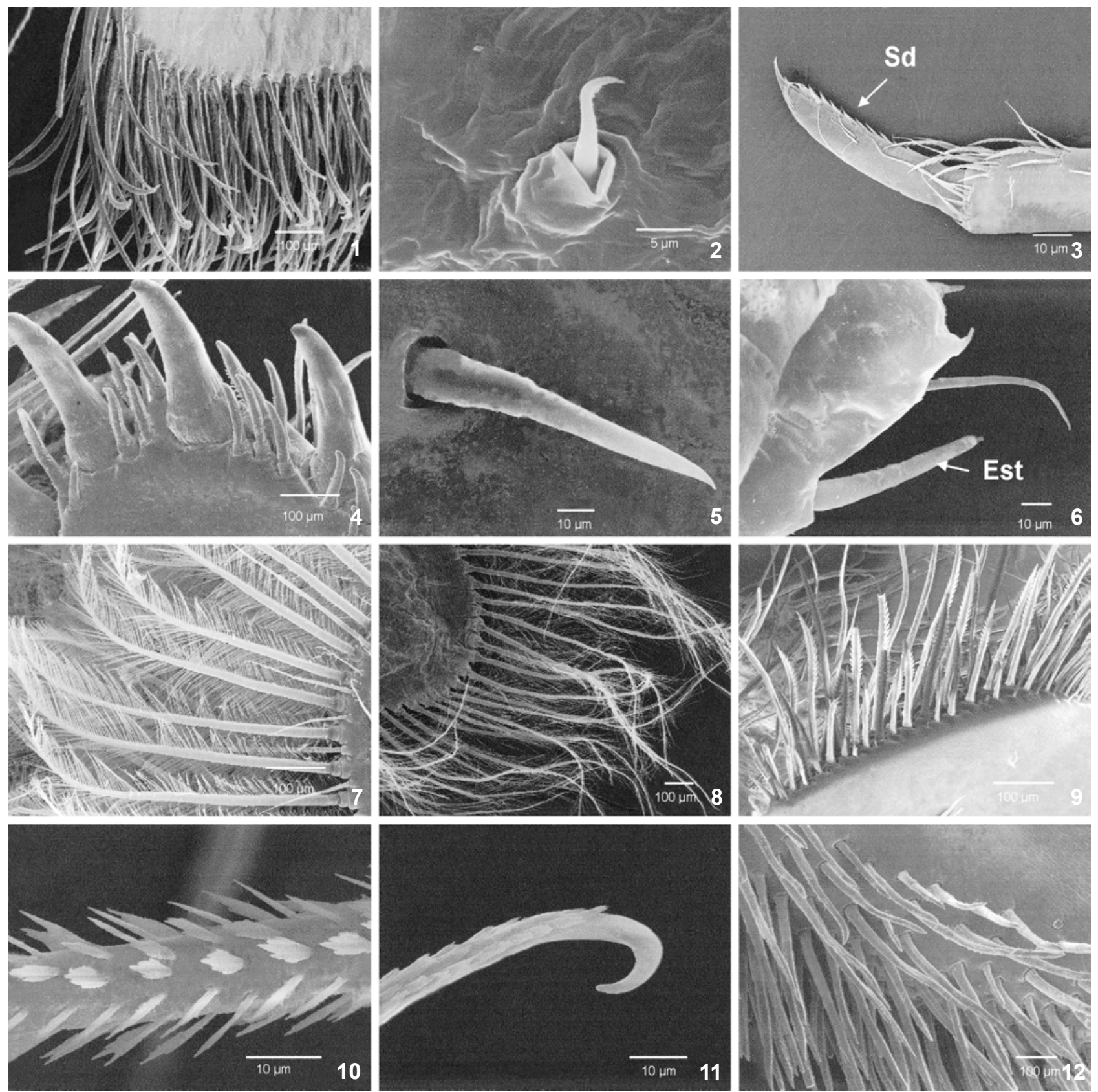

Figuras 1-12. Setas de Parastacus brasiliensis: (1) setas simples, vista ventral do basipodito da segunda maxila; (2) seta gancho, superfície do epipodito do primeiro maxilípodo; (3) seta dentiforme (Sd), margem ventral do dactilopodito do quarto pereiópodo; (4) setas cuspidadas, região apical do dactilopodito do segundo maxilípodo; (5) seta conada, superfície do escafognatito da segunda maxila; (6) estetasco (Est), margem apical da região distal do flagelo externo da primeira antena; (7) setas plumosas, margem do telso; (8) setas paposas, margem apical do escafognatito da segunda maxila; (9) setas serradas, vista ventral da região basal oral do isquiopodito do terceiro maxilípodo; (10) seta multidenticulada, basipodito da primeira maxila em sua margem aboral na região médio distal; (11) setobrânquia, região dorsal do coxopodito do terceiro pereiópodo; (12) setas "teazel", artículo distal do palpo mandibular. 
em todas as setas examinadas. Septo basal presente. 12) Setobrânquias (Fig. 11): forma similar àquela da seta multidenticulada; muito longa e afilada (7-8 mm de comprimento). Anulação evidente, próximo à metade da haste. Dentículos ocorrem logo após a anulação e apresentam projeções na região distal. Ápice da setaa fino, liso e recurvado. 13) Seta "Teazel" (Fig. 12): anulação evidente na região mediana da haste, com dentículos alongados após a anulação.

\section{DISCUSSÃO}

As setas de $P$. brasiliensis, assim como o observado por Tномаs $(1970,1973)$ em Austropotamobius pallipes, apresentam uma grande variedade de tipos morfológicos e padrões de distribuição pela superfície dos apêndices.

Existem setas que se encontram amplamente distribuídas nos apêndices e que apresentam variações de comprimento conforme a localização, como as setas simples, as setas serradas e as setas paposas; setas com distribuição restrita e de tamanho uniforme, como as setas gancho, os estetascos, as setas dentiformes e as setas conadas. No dactilopodito do segundo maxilípodo, ocorre um tipo especial de seta cuspidada com dentículos, que se encontra associada a setas cuspidadas lisas e maiores, bem como no estatocisto da $1^{\text {a }}$ antena, onde existem tipos especiais de setas paposas, concordando com as observações de Thomas (1970) em A. pallipes.

Apesar de se tratar de um lagostim europeu da família Astacidae, o padrão de distribuição das setas de A. palliles descrito por Thомаs (1970) é semelhante ao encontrado em $P$. brasiliensis. Entretanto, algumas diferenças foram constatadas, como a ausência de setas serradas (chamadas de serruladas por Thomas (1970) no basipodito e coxopodito do primeiro maxilípodo e primeira maxila, onde foram encontradas setas plumodenticuladas e multidenticuladas. Outra diferença encontrada foi a presença de setas multidenticuladas no basipodito da primeira maxila, que não foram mencionadas por aquele autor. Um fato a ser considerado, é que THomas (1970) fez suas descrições baseadas em microscopia óptica, diferentemente das que foram feitas no presente trabalho nós, utilizando microscopia eletrônica de varredura.

\section{REFERÊNCIAS BIBLIOGRÁFICAS}

Bond-Buckup, G.; A.A.P. Bueno \& K.A Keunecke. 1996. Primeiro estágio juvenil de Aegla prado Schmitt (Crustacea, Decapoda, Anomura, Aeglidae). Revista Brasileira de Zoologia, Curitiba, 13 (4): 1049-1061.

- 1998. Morphological characteristics of juvenile specimens of Aegla (Decapoda, Anomura, Aeglidae). Proceedings of the Fourth Internacional Crustacean Congress, Amsterdam, 1: 371-381.

Buckup, L. 1999. Família Parastacidae (lagostins-de-água-doce), p. 319-327. In: L. BUCKUP \& G. BOND-BUCKUP (Eds). Os crustáceos do Rio Grande do Sul. Porto Alegre, Editora UFRGS, 503p.
Buckup, L. \& A. Rossı. 1980. O gênero Parastacus no Brasil (Crustacea, Decapoda, Parastacidae). Revista Brasileira de Biologia, Rio de Janeiro, 40 (4): 663-681.

Bueno, A.A.P. \& G. Bond-Buckup. 1996. Os estágios iniciais de Aegla violacea Bond-Buckup \& Buckup (Crustacea, Anomura, Aeglidae). Nauplius, Botucatú, 4: 39-47.

CALAZANS, D.\& R. InGLE. 1998. The setal morphology of the larval phases of the Argentinean red shrimp Pleoticus muelleri Bate, 1888 (Decapoda: Solenoceridae). Invertebrate Reproduction and Development, London, 33 (2-3): 109-126.

Drach, P. \& F. Jacques. 1977. Système sétifère des crustacés décapodes: Principes d'une classification générale. Compte Rendus de la Academie des Sciences, Paris, 284: 1995-1998.

FARMER, A.S. 1974. The functional morphology of the mouthparts and pereiopods of Nephrops norvegicus (L.) (Decapoda: Nephropidae). Journal of Natural History, London, 8: 121142.

Felgenhauer, B.E. 1987. Techniques for preparing crustaceans for scanning eletron microscopy. Journal of Crustacean Biology, Kansas, 7 (1): 71-76.

FISH, S. 1972. The setae of Eurydice pulchra (Crustacea: Isopoda). Journal of Zoology, London, 166: 163-177.

FonTOURA, N.F. \& L. BucKup. 1989a. O crescimento de Parastacus brasiliensis (von Martens, 1869) (Crustacea, Decapoda, Parastacidae). Revista Brasileira de Zoologia, Curitiba, 49 (4): 897-909.

. 1989b. Dinâmica populacional e reprodução em Parastacus brasiliensis (von Martens, 1869) (Crustacea, Decapoda, Parastacidae). Revista Brasileira de Zoologia, Curitiba, 49 (4): 911-921.

JACQUES, F. 1989. The setal system of crustaceans: types of setae, groupings, and functional morphology, p.1-13. In: B.E. Felgenhauer; L. Watling \& A.B. Thistle (Eds). Functional morphology of feeding and grooming in Crustacea. Crustacean Issues 6. Rotterdam, A.A. Balkema, X+225p.

ScotTo, L.E. 1980. Studies on Decapod Crustacea from the Indian River Region of Florida. XIV: A Method for rapid preparation of Brachyuran larval for scanning electron microscopy. Crustaceana, Leyden, 38 (1): 99-101.

Thомаs, W.J. 1970. The setae of Austropotamobius pallipes (Crustacea: Astacidae). Journal of Zoology, London, 160, 91-142.

. 1973. The hatchling setae of Austropotamobius pallipes Lereboullet) (Decapoda, Astacidae). Crustaceana, Leyden, 24: 77-89.

WatLING, L. 1989. A classification system for crustacean setae based on the homology concept, p. 15-26. In: B.E. FeLGENHAUER; L. WATLING \& A.B. ThistLe (Eds). Functional morphology of feeding and grooming in Crustacea. Rotterdam, A.A. Balkema, Crustacean Issues 6, X+225p.

Recebido em 02.II.2004; aceito em 16.IX.2004. 